Utz L, ${ }^{1}$ - Peresini V, ${ }^{2}$

Farías $A,{ }^{3}$ - Gauna D, ${ }^{4}$

Peisino $M,^{5}$ - Almada $D,{ }^{6}$ Acevedo $\mathrm{G},{ }^{7}$

${ }^{1}$ Magister en Salud Sexual y Reproductiva., Profesor adjunto de la Cátedra de Medicina Preventiva y Social Facultad de Ciencias Médicas de la Universidad Nacional de Córdoba. Mail:lisandroutz@yahoo. com arORCID ID 0000-000-2-36290424. Dirección postal Hospital Nacional de Clínicas Santa Rosa 1564 5000 Córdoba teléfono 3514337014

${ }^{2}$ Médica, Especialista en Medicina

Familiar. Profesor asistente de la

Cátedra de Medicina Preventiva y Social Facultad de Ciencias Médicas de la Universidad Nacional de Córdoba. № de investigadora: 972. Mail:virperesini81@hotmail.com. ${ }^{3}$ Magister en Salud Pública, Profesora adjunta de la Cátedra de Medicina Preventiva y Social Facultad de Ciencias Médicas de la Universidad Nacional de Córdoba. Investigadora del Centro de Investigación y Formación en Gestión de Servicios de Salud y Trabajo (CeGeSST), de la Escuela de Salud Pública, Facultad de

Ciencias Médicas de la Universidad

Nacional de Córdoba. Mail:afarias@ fcm.unc.edu.arORCID ID 0000-00034037-5969

4Doctor en Salud Pública, Profesor Asistente dela Cátedra de Medicina Preventiva y Social Facultad de Ciencias Médicas de la Universidad Nacional de Córdoba. Mail: diegogauna05@gmail.com.

${ }^{5}$ Médica, Especialista en Medicina

Familiar. Profesor asistente de la

Cátedra de Medicina Preventiva y Social Facultad de Ciencias Médicas de la Universidad Nacional de Córdoba. Mail: eugepeisino@hotmail. com ${ }^{6}$ Médico Pediatra, Profesor Asistente dela Cátedra de Medicina Preventiva y Social Facultad de Ciencias Médicas de la Universidad Nacional de Córdoba. Mail: diegoalmadab@ gmail.com.

'Doctor en Medicina y Cirugía,

Profesor titular de la Cátedra de Medicina Preventiva y Social Facultad de Ciencias Médicas de la Universidad Nacional de Córdoba. Director del Centro de Investigación y Formación en Gestión de Servicios de Salud y Trabajo (CeGeSST), de la Escuela de Salud Pública, Facultad de Ciencias Médicas de la Universidad Nacional de Córdoba. Mail: gabrieleacevedo@ gmail.com

Trabajo recibido: 03 de abril 2018.

Aprobado: 10 de octubre

\section{MOTIVOS DE CONSULTA PREVALENTES EN CENTROS DE SALUD DE PRIMER NIVEL DE ATENCIÓN DE LA CIUDAD DE CÓRDOBA, AÑO 2016.}

\author{
PREVAILING CONSULTATION REASONS AT \\ FIRST LEVEL HEALTH CARE CENTERS IN \\ THE CITY OF CORDOBA, YEAR 2016.
}

\section{RAZÕES PARA CONSULTA PREVALENTE EM CENTROS DE SAÚDE DE CUIDADOS DO PRIMEIRO NÍVEL NA CIDADE DE CÓRDOBA, ANO 2016.}

\section{Resumen}

El objetivo fue identificar y analizar los motivos de consulta prevalentes en los centro de salud de primer nivel de atención de la Municipalidad de Córdoba durante el 2016 con el propósito de adecuar y optimizar la oferta sanitaria. Estudio descriptivo, transversal, se analiza la totalidad de las consultas de primera vez en 97 centros. La base de datos fueron atenciones médicas registradas según Clasificación Internacional de Enfermedades (CIE10). De las 500.071 consultas totales, $61,25 \%$ fueron realizadas por mujeres. El 49,6\% correspondió a controles de salud, siendo el del niño el que presentó mayor registro en ambos sexos. Respecto a los diez principales motivos de consulta, la patología aguda de las vías respiratorias presentó mayor demanda en ambos sexos. El registro de problemas psicosociales fue bajo, no se encontraron registros de factores de riesgo 
cardiovascular. Conocer el perfil de demanda promueve una mayor adecuación en la oferta de servicios.

Palabras clave: Servicios de salud/utilización - atención primaria de salud - atención ambulatoria - epidemiología.

\section{Abstract}

The objective is to identify and analyze prevailing consultation reasons at first level health care centers from the Municipality of Cordoba during the year 2016 in order to adequate and optimize the health offer. Descriptive, cross sectional study; all first time consultations are analyzed. The data bases used were medical consultations, recorded according to the International Classification of Diseases (ICD10). From the 500,001 total consultations, $61.25 \%$ weredone by women. The $49.6 \%$ corresponded to health check ups; with children showing higher records in both sexes. Regarding ten main reasons of consultation, acute respiratory tract condition was the most frequent in both sexes. Few psychosocial problems were recorded and there were no records or cardiovascular risk. Being aware of the demand profile, fosters a greater adequacy of the services offered.

Key words: Health services/use - primary health care - ambulatory care - epidemiology.

\section{Resumo}

O objetivo é identificar e analisar as razões para consulta prevalente no centro de saúde de cuidados primários do Município de Córdoba, durante 2016, a fim de se adaptar e otimizar o fornecimento de cuidados de saúde. Pesquisa descritiva, transversal, analisando a totalidade das primeiras consultas em 97 centros. O banco de dados é baseado em atendimentos médicos registrados de acordo com a Classificação Internacional de Doenças (CIE10). Do total de 500.071 consultas, $61,25 \%$ foram realizadas por mulheres. $49,6 \%$ corresponderam a controles de saúde, tendo as crianças o maior registro em ambos os sexos. Em relação com os dez principais motivos de consulta, a patologia aguda do trato respiratório apresentou maior demanda em ambos os sexos. O registro de problemas psicossociais foi baixo, não foram encontrados registros de fatores de risco cardiovascular. Conhecer o perfil da demanda promove maior adequação na oferta de serviços.

Palavras chave: Serviços de saúde / utilização - atenção primária à saúde - atendimento ambulatorial - epidemiologia.

\section{Introducción}

El adecuado conocimiento de los problemas de salud de una comunidad resulta esencial para determinar el tipo de actividades de atención (promoción, prevención y asistencia); asignar recursos en cantidad y calidad conforme a las necesidades poblacionales y evaluar el grado de adecuación de la respuesta del sistema sanitario con el objeto de implementar las correcciones necesarias para brindar un mejor servicio de salud a la población ${ }^{1}$. En este sentido, el análisis del uso de los servicios de salud de los sistemas públicos y sus determinantes es clave para evaluar el acceso e identificar desigualdades sociales en la utilización. La influencia de los distintos determinantes de la utilización varía según el nivel asistencial y el país ${ }^{2}$.

En la ciudad de Córdoba, Argentina, reside una población de más de 1.4 millones de habitantes, entre los cuales aproximadamente el $35 \%$ no posee cobertura de salud de los subsistemas de la seguridad social o privados, y por ello tienen como principal fuente de atención a los servicios de salud dependientes de la Municipalidad de Córdoba, en especial en lo que al primer nivel de atención se refiere ${ }^{3}$. 
La estrategia de atención primaria de la Salud (APS) se plantea como el eje estructurador y organizador del sistema y los servicios de salud. Clásicamente se distinguen tres niveles de atención. El primer nivel es el más cercano a la población, o sea es la puerta de entrada al sistema de salud. Se espera que un equipo de APS bien conformado resuelva entre el 80 y $95 \%$ de los problemas de salud de la población. Según Starfield, apenas 26 problemas de salud involucran al $50 \%$ de todos los motivos de consulta de médicos de familia en Estados Unidos ${ }^{4}$.

Este nivel permite una adecuada accesibilidad a la población, pudiendo realizar una atención oportuna y eficaz ${ }^{5}$.El sistema de Salud Municipal está organizado en 6 zonas sanitarias y brinda asistencia a través de una red de servicios en los tres niveles de atención. El primer nivel de atención está compuesto por 97 centros de salud distribuidos por todo el ejido municipal. En estos centros se brinda atención a la demanda espontánea y se llevan a cabo programas de salud normatizados, como el control de salud del niño, de la embarazada, del adolescente, del adulto, salud sexual y reproductiva (SSyR) y programas específicos para problemas de salud como hipertensión arterial, diabetes, tuberculosis e infecciones de transmisión sexual. El recurso humano que se desempeña en el primer nivel de atención está conformado por 1.129 trabajadores (profesionales, técnicos y administrativos y auxiliares).

La población que demandan servicios en el primer nivel de atención público de la ciudad de Córdoba, son predominantemente mujeres y niños/as, con una proporción de adultos mayores inferior a la de la media de la población de la ciudad. Frecuentemente pertenecen a hogares de bajos ingresos, con bajo nivel de escolarización y con empleo informal ${ }^{6}$.

Se reconoce que la demanda de atención de salud se encuentra condicionada por factores relacionados con el perfil epidemiológico de la población y con las características de los servicios ofrecidos.

Es así que se han postulado tres tipos de características que determinan el uso (y no uso) de servicios ambulatorios del primer nivel de atención, en especial de servicios de dependencia estatal: características sociodemográficas de individuos y hogares, algunas características a nivel localidad relacionadas con la existencia de servicios y niveles de marginalidad, $\mathrm{y}$, finalmente, los perfiles de protección social de los individuos? ${ }^{7}$.

El objetivo del trabajo fue identificar y analizar los motivos de consulta prevalentes en los centro de salud de primer nivel de atención dependientes de la Municipalidad de Córdoba durante el período 2016 con el propósito de generar información que permita sustentar decisiones orientadas a lograr una optimización de los servicios de salud ofrecidos y mayor adecuación a la demanda de atención.

\section{Material y Método}

A fin de cumplimentar con el objetivo de esta investigación se diseñó un estudio de tipo descriptivo, transversal, que analiza la totalidad de las consultas atendidas por primera vez en los 97 centros de salud dependientes del municipio de Córdoba durante el año 2016. La fuente de información fue la base de datos de los registros de atención médica diaria cuyos diagnósticos se encontraban clasificados según Clasificación Internacional de Enfermedades (CIE10).Conforme a esta clasificación, los problemas de salud fueron agrupados en diferentes categorías. En la categoría problemas respiratorios agudos se incluyeron los problemas de la vía respiratoria superior e inferior (rinitis, faringitis, laringitis, bronquitis, neumonía); en problemas agudos digestivos se agruparon las consultas por diarreas, vómitos y dolor abdominal; en problemas en piel se incluyó a las dermatitis e infecciones (piodermititis, impétigo, forunculosis, abscesos, escabiosis, etc.) y en problemas respiratorios crónicos asma y enfermedad pulmonar obstructiva crónica (EPOC). Además, se agrupó en la categoría trastornos del ciclo menstrual las consultas por amenorreas, irregularidad menstrual e hipermenorrea y en problemas agudos de oído 
(otalgia y otitis). En la categoría correspondiente a traumatismos se incluyó a los accidentes, heridas y caídas. Los dolores del sistema osteomioarticular (SOMA) considerados dentro de este grupo fueron: lumbagos, lumbago con ciática o ciatalgia, dorsalgias, cervicalgias, etc. El resto de los motivos de consulta, tales como fiebre, hipertensión (HTA), obesidad y sobrepeso, Diabetes (DBT), infecciones del tracto urinario (ITU) y vulvovaginitis se consignaron individualmente.

Las variables dependientes fueron las consultas registradas y la independiente el sexo de las personas atendidas.

Se aplicaron análisis descriptivos calculando medidas de frecuencias absolutas y relativas según tipo de consultas y sexo. Para estos análisis se aplicó el software estadístico InfoStat v.2012.

\section{Resultados}

Durante el año 2016 se registró un total de 500.071 consultas reportadas como atenciones de primera vez en los 97 centros de salud. De estas consultas $306.298(61,25 \%)$ fueron realizadas por mujeres y $193.773(38,75 \%)$ por varones. Un total de 247.988 consultas $(49,6 \%)$ correspondieron a atenciones relacionadas a controles de salud como certificados médicos, control de salud del niño, adolescente, adulto, embarazo y salud sexual y reproductiva y 252.083 consultas se registraron como problemas de salud (Figura 1).

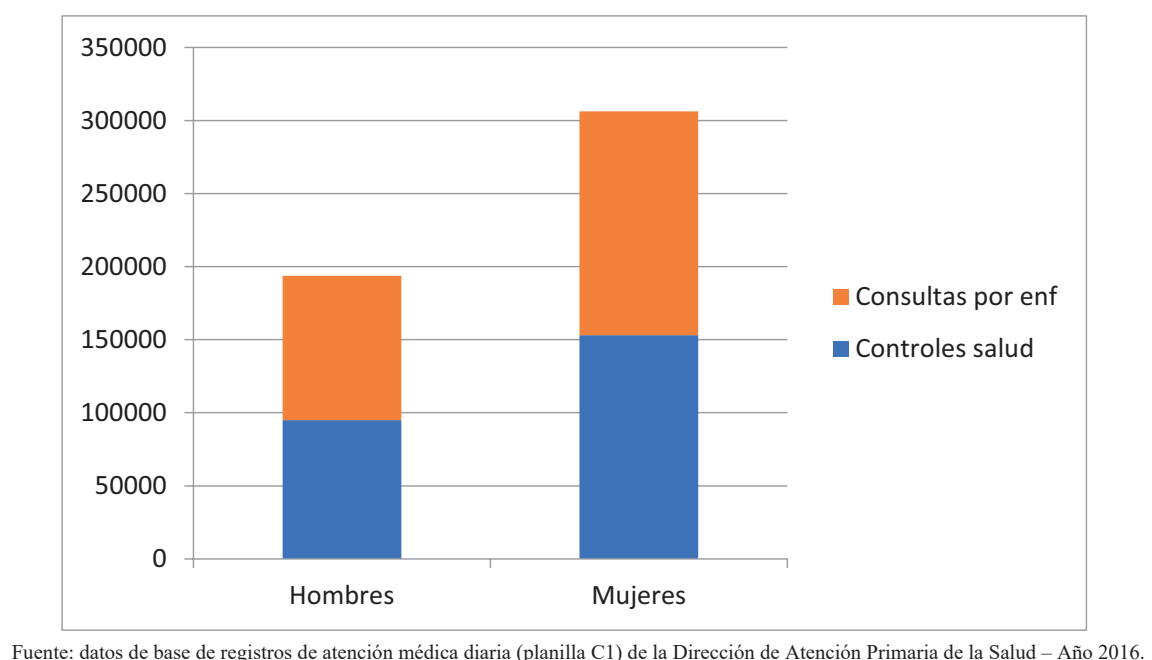

Respecto a las consultas por controles de salud, en los hombres, el control del niño representa el 51,4\% del total de estas consultas. En cambio, en las mujeres, las consultas relacionadas a controles de salud se distribuyen de manera dispar, representando los controles del niño el $28 \%$ de las consultas por controles de salud, el control de embarazo y puerperio el 14,3\% ; salud sexual y reproductiva y control ginecológico el 23,3\% de las consultas (Tabla 1).

Figura No1: Distribución de consultas según sexo y tipo de consulta, año 2016. 
Tabla Nº1: Motivos de consultas relacionados a controles de salud según sexo, año 2016.

\begin{tabular}{|c|c|c|c|c|c|}
\hline \multicolumn{3}{|c|}{ Hombres } & \multicolumn{3}{|c|}{ Mujeres } \\
\hline $\begin{array}{c}\text { Control de } \\
\text { Salud }\end{array}$ & $\begin{array}{c}\mathbf{N}^{\circ} \\
\text { consultas } \\
\end{array}$ & \begin{tabular}{|c|}
$\%$ de las \\
consultas \\
por controles \\
de salud \\
\end{tabular} & $\begin{array}{c}\text { Control de } \\
\text { salud }\end{array}$ & $\begin{array}{c}\mathbf{N}^{\circ} \\
\text { consultas }\end{array}$ & $\begin{array}{c}\% \text { de las } \\
\text { consultas por } \\
\text { controles de } \\
\text { salud } \\
\end{array}$ \\
\hline Niño & 48812 & 51,4 & Niño & 42908 & 28 \\
\hline Adolescente & 4080 & 4,3 & Adolescente & 4746 & 3,1 \\
\hline Adulto & 19177 & 20,2 & Adulto & 21772 & 14,2 \\
\hline $\begin{array}{l}\text { Salud Sexual } \\
\text { y } \\
\text { Reproductiva }\end{array}$ & 0 & 0 & $\begin{array}{l}\text { Embarazo y } \\
\text { Puerperio }\end{array}$ & 21846 & 14,3 \\
\hline & & & 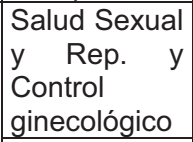 & 35582 & 23,3 \\
\hline $\begin{array}{l}\text { Certificado } \\
\text { médico }\end{array}$ & 22846 & 24,1 & $\begin{array}{l}\text { Certificado } \\
\text { médico }\end{array}$ & 26219 & 17,1 \\
\hline Total & 94915 & 100 & & 153073 & 100 \\
\hline
\end{tabular}

Fuente: datos de base de registros de atención médica diaria (planilla C1) de la Dirección de Atención Primaria de la Salud - Año 2016.

En relación al total de las consultas registradas como problemas de salud, el 58\% de las mismas fueron realizadas por mujeres.

Respecto ala distribución de los 10 principales motivos de consulta registrados, se observan variaciones de acuerdo al sexo.

En ambos sexos, el motivo de consulta que presentó mayor demanda de servicios de salud fue las relacionadas a síntomas agudos de vías respiratorias. Además, en ambos sexos se registraron problemas agudos de oído, de piel, gastrointestinales, dolor del sistema osteomioarticular y fiebre. En las mujeres, se objetivan además las infecciones urinarias y vulvovaginitis. A diferencia de estas, en los hombres aparecen los accidentes debido a externalidades dentro de los principales motivos de consultas.

En relación a los problemas de salud que involucran a enfermedades crónicas no transmisibles (ECNT), la HTA y la DBT se encuentran dentro de los primeros motivos de consulta en ambos sexos, en hombres se registran además los problemas respiratorios crónicos, el sobrepeso y obesidad.No se encontraron registros de atención por factores de riesgo cardiovascular como tabaquismo, dislipemia, sedentarismo, etc.

Estos motivos de consulta incluidos en las diez categorías anteriormente descriptas representan en hombres el $83,2 \%$, y en mujeres el $75,1 \%$ de la totalidad de las consultas por problemas de salud del primer nivel de atención(Tabla 2).

Tabla N²: Principales motivos de consulta por problemas de salud según sexo, año 2016.

\begin{tabular}{|c|c|c|c|c|c|}
\hline \multicolumn{3}{|c|}{ Hombre } & \multicolumn{3}{|c|}{ Mujer } \\
\hline Motivo & $\begin{array}{c}\mathrm{N}^{\circ} \text { de } \\
\text { consultas }\end{array}$ & $\begin{array}{c}\% \text { de las } \\
\text { consultas por } \\
\text { problemas de } \\
\text { salud }\end{array}$ & Motivo & $\begin{array}{c}\mathrm{N}^{\circ} \mathrm{de} \\
\text { consultas }\end{array}$ & $\begin{array}{c}\% \text { de las } \\
\text { consultas por } \\
\text { problemas de } \\
\text { salud }\end{array}$ \\
\hline $\begin{array}{l}\text { Agudas } \\
\text { Respiratorias }\end{array}$ & 51319 & 51,9 & $\begin{array}{l}\text { Agudas } \\
\text { Respiratorias }\end{array}$ & 60672 & 40,2 \\
\hline \begin{tabular}{|l} 
Agudas \\
digestivas
\end{tabular} & 5700 & 5,8 & $\begin{array}{l}\text { Hipertensión } \\
\text { Arterial }\end{array}$ & 9050 & 6 \\
\hline $\begin{array}{l}\text { Hipertensión } \\
\text { arterial }\end{array}$ & 4994 & 5,1 & Agudas digestivas & 8053 & 5,3 \\
\hline Problemas Piel & 4797 & 4,9 & Problemas Piel & 6425 & 4,3 \\
\hline Fiebre & 2784 & 2,8 & $\begin{array}{l}\text { Alteración del ciclo } \\
\text { menstrual }\end{array}$ & 5.586 & 3,7 \\
\hline Agudas oído & 2693 & 2,7 & $\begin{array}{l}\text { Infección del tracto } \\
\text { urinario }\end{array}$ & 5075 & 3,4 \\
\hline
\end{tabular}




\begin{tabular}{|l|c|c|l|c|c|} 
Diabetes & 2455 & 2,5 & $\begin{array}{l}\text { Dolor sistema } \\
\text { osteomioarticular }\end{array}$ & 4377 & 2,9 \\
\hline Traumatismos & 2193 & 2,2 & Diabetes & 4170 & 2,8 \\
\hline $\begin{array}{l}\text { Crónicas } \\
\text { respiratorias }\end{array}$ & 1948 & 2 & Vulvovaginitis & 3583 & 2,4 \\
\hline $\begin{array}{l}\text { Dolor sistema } \\
\text { Osteomioarticular }\end{array}$ & 1891 & 1,9 & Agudas Oído & 3397 & 2,2 \\
\hline $\begin{array}{l}\text { Obesidad- } \\
\text { Sobrepeso }\end{array}$ & 1433 & 1,4 & Fiebre & 2898 & 1,9 \\
\hline Total & 82207 & 83,2 & Total & 113286 & 75,1 \\
\hline
\end{tabular}

Fuente: datos de base de registros de atención médica diaria (planilla C1) de la Dirección de Atención Primaria de la Salud - Año 2016

En lo que refiere al registro de problemas psicosociales tales como, ansiedad, depresión, consumo de sustancias o violencia en el primer nivel de atención, se encontró que del total de consultas por problemas de salud estos representan solo el 0,2\% (n: 216) en hombres y del 0,4\% (n: 611) en mujeres (Tabla 3).

Tabla No3: Distribución de motivos de consulta por problemas psicosociales según sexo, año 2016.

\begin{tabular}{|l|l|l|}
\hline \multicolumn{1}{|c|}{ Trastornos psicosociales } & \multicolumn{1}{|c|}{\begin{tabular}{c}
\multicolumn{1}{c|}{$\mathbf{N}^{\circ}$ consultas } \\
Hombres
\end{tabular}} & \multicolumn{1}{c|}{\begin{tabular}{c}
\multicolumn{1}{c|}{ No consultas } \\
Mujeres
\end{tabular}} \\
\hline $\begin{array}{l}\text { Consumo de sustancias y } \\
\text { alcohol }\end{array}$ & 35 & 21 \\
\hline Ansiedad & 151 & 443 \\
\hline Depresión & 29 & 131 \\
\hline Violencia & 1 & 16 \\
\hline Total & 216 & 611 \\
\hline Fuente: datos de base de registros de atención médica diaria (planilla C1) de la Dirección de Atención Primaria de la Salud - Año 2016
\end{tabular}

\section{Discusion}

Conforme a los resultados obtenidos en el presente estudio, las consultas por control de salud representan en ambos sexos el principal motivo de consulta en el primer nivel de atención. Por otro lado, las enfermedades respiratorias agudas ocupan el primer lugar dentro de los motivos de consulta por problema de saluden ambos sexos. Comparando estos datos con otros registros de consultas del primer nivel, los problemas respiratorios agudos ocupan también el primer lugar. El resto de los motivos de consultas registrados como los prevalentes en el presente estudio se corresponden con los registrados en el primer nivel de atención de la Ciudad Autónoma de Buenos Aires (CABA) ${ }^{8}$ y con los reportados en una red de atención primaria en Argentina?.

Respecto a las consultas por ECNT, el porcentaje que representan éstas se asemejan a los reportados en otros de los estudios realizados en Argentina, constituyendo aproximadamente un $5 \%$ de las consultas por problemas de salud.

A diferencia de otros registros del primer nivel de atención, en este estudio, los problemas psicosociales como ansiedad, depresión, consumo de sustancias o violencia, constituyen un mínimo porcentaje del total de las consultas, lo que podría deberse a que las personas consultantes no los perciben como problemas que requieran atención médica y/o a que el profesional médico no los registra como principal motivo de consulta.

Teniendo en cuenta el fenómeno de transición epidemiológica que atraviesa la Argentina, resulta necesario investigar las causas que traen aparejado un bajo registro de las patologías crónicas y sus factores de riesgo en el primer nivel en el primer nivel de atención. Si la misma se debe a una reducida demanda, una limitada oferta o a una falta de sensibilización respecto a la importancia de su registro. A su vez, la baja demanda podría estar relacionada a un desconocimiento de la población sobre la competencia del primer nivel de atención 
para el abordaje de esta problemática o a la ausencia de problematización de factores de riesgo como la obesidad, el tabaquismo o consultas de la esfera psicosocial como adicciones, violencia , ansiedad, depresión, entre otras.

En síntesis, del estudio realizado surge que el perfil de consultas atendidas en los centros de primer nivel de atención públicos de la ciudad de Córdoba durante el año 2016 se caracteriza por una similar distribución cuantitativa entre controles de salud y las referidas a la atención de problemas de salud, siendo estos últimos predominantemente por afecciones agudas. El reconocimiento de este perfil puede dar sustento a acciones sanitarias orientadas a lograr un mayor nivel de adecuación entre los requerimiento de atención de la población usuaria d estos servicios y los que se ofrecen efectivamente.

\section{Bibliografía}

1. Acevedo G, Martínez D, UtzL. La salud y sus determinantes, la salud pública y la medicina preventiva.En: Acevedo G, Manual de Medicina Preventiva y Social I. Facultad de Ciencias Médicas, Universidad Nacional de Córdoba. Edición ACSIDAS, Año 2012. Pag. 5-20.

2. Garcia I, Vargas I,Amparo L, Mogollón S,PérezC, De Paeped P y col. Determinantes del uso de distintos niveles asistenciales en el Sistema General de Seguridad Social en Salud y Sistema Único de Salud en Colombia y Brasil. Gaceta Sanitaria.2014; 28, (6): 480-488.

3. Instituto Nacional de Estadística y Censos Censo nacional de población, hogares y viviendas 2010. Buenos Aires: Instituto Nacional de Estadística y Censos - INDEC, 2012. [Citado el 10 de Diciembre de 2017]. Disponible en: https://www.indec.gov. $\mathrm{ar} / \mathrm{ftp} /$ cuadros/poblacion/censo2010 tomo1.pdf

4. Araújo Porto G, Cançado L, Bonamigo A, Miranda Rocha J, Le Sann Nascimento J, Azagra R. Análise de demanda em Medicina de Família no Brasil utilizando a Classificação Internacional de Atenção Primária.Ciênc. saúde coletiva [Internet].2012 . [Citado el 10 de Diciembre de 2017]; 17 (11). Disponible en: http:// www.scielosp.org/scielo.php?script=sci_arttext\&pid=S1413-81232012001100019

5. Vignolo J. Vacarezza M. Álvarez C. Sosa A. Niveles de atención, de prevención y atención primaria de la salud. ArchMed Interna 2011; XXXIII (1):11-14.

6. Secretaría de Salud, Municipalidad de Córdoba. Perfil epidemiológico de la población de la ciudad de Córdoba, año 2015. [Citado el 7 de Diciembre de 2017]. Disponible en: www.gobiernoabierto.cordoba.gob.ar/data/datos-abiertos/categoria/ salud/demografia-sanitaria/144.

7. Bautista-Arredondo S, Serván-Mori E, Colchero M, Ramírez-Rodríguez B, SosaRubí S, Análisis del uso de servicios ambulatorios curativos en el contexto de la reforma para la protección universal en salud en México. Salud pública Méx. 2014 ; 56(1): 18-31

8. Gobierno de la Ciudad de Buenos Aires, Ministerio de Salud. Sistema de Información de Centros de Salud [Internet]. Buenos Aires. 2011. [Citado el 10 de Diciembre de 2017]; Síntesis del año 2011 centros de salud y cobertura porteña desalud-plan médico de cabecera. Disponible en: http://www.buenosaires.gob.ar/ salud/estadisticas-para-la-salud/atencion-primaria-de-la-salud

9. Rubinstein A. KopitowskiK. Bases y Fundamentos de la Práctica de la Medicina Familiar en: Karin Kopitowski y col. Medicina Familiar y Práctica Ambulatoria. $3^{\circ}$ Edición. Ciudad Autónoma de Buenos Aires Editorial. Panamericana.2016. Pág 12 al 14 